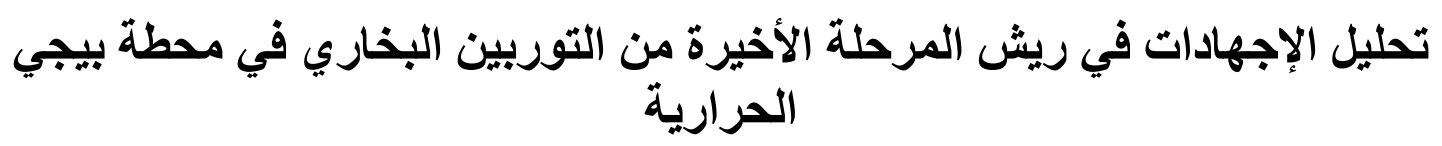

\author{
الاء محمد عبدالله \\ جامعة الموصل/ كلية الهندسة / قسم الهندسة الميكانيكية
}

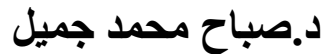 \\ استاذ / جامعة الموصل /كلية الهندسة / قسم الهندسة الميكاتيكية
}

\title{
الخلاصة
}

تعاني ريش التوربين البخاري وخاصة الطويلة تأريخ من الفنشل لايمكن تحديد سببها بدقة لكثرة المتغيرات

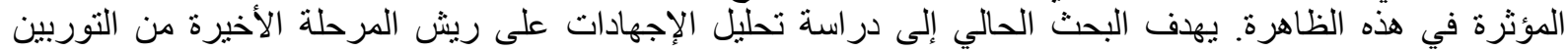

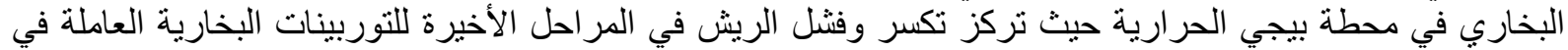

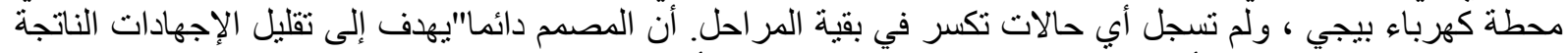

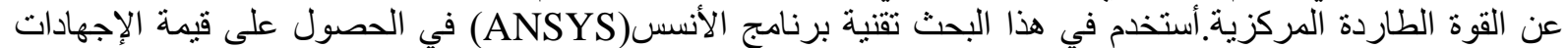

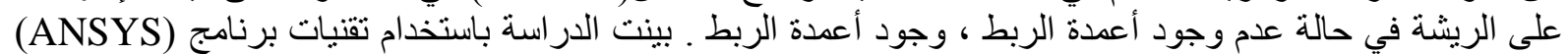

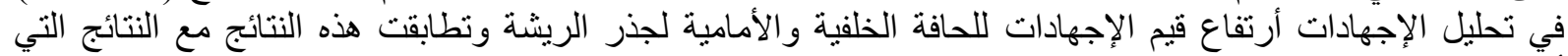

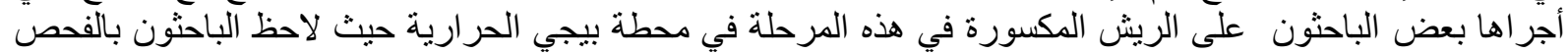

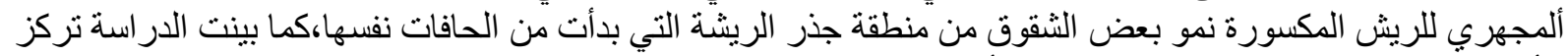
الأجهادات في النصف الرئوي اللورة للريشة وحول أعمدة الربط.

الكلمات المفتاحية : التوربين البخاري، الإجهاد، التصميم

\section{Stress Analysis of Steam Turbine Blades at The last Stage in Beji Thermal power Station}

\section{Dr. Sabah M.J. Ali}

Alaa M. Abdullah
Univ. of Mosul/College of Eng. /Mechanical Dep.

Prof./Univ. of Mosul/College of Eng./Mechanical Dep.

\begin{abstract}
The low pressure steam Turbine blades are suffering especially the longest parts a bad history of failure. The breakage reasons are unknown due to its complexity. The aim of this work concentrated for the analysis of stresses at the last stage blades in Baji thermal power station. No records have been recorded of breakage incident at any other stages. The aim of the designer is to find way of reducing stresses due to centrifugal force.(ANSYS 12.1) is used for obtaining various stresses in case. The blades without lacing rod the blades with lacing rod. It was concluded throughout using (ANSYS) Techniques, analysis increasing stresses at the leading and trailing edge of blade root and a stress concentration at the upper mid of the blade. This indicates why a failure at the lacing rod and aerofoil occurred in this station.
\end{abstract}




\section{قائمة الرموز}

\begin{tabular}{|c|c|c|}
\hline 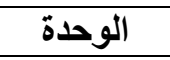 & المعنى & 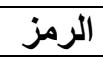 \\
\hline $\mathrm{kg}$ & كتلة الريشة & $\mathrm{m}$ \\
\hline $\mathrm{m}$ & طول الريشة & $\mathrm{Lb}$ \\
\hline$m^{2}$ & مساحة مقطع الريشة & A \\
\hline $\mathrm{rad} / \mathrm{sec}$ & السرعة الزاوية & $\omega$ \\
\hline $\mathrm{N}$ & القوة الطاردة المركزية & $F_{c f}$ \\
\hline $\mathrm{m}$ & نصف قطر عمود الدوران & $R_{r}$ \\
\hline$m^{2}$ & مساحة مقطع الريشة عند الجذر & $A r$ \\
\hline$m^{2}$ & مساحة مقطع الريشة العلوي & At \\
\hline $\mathrm{m}$ & أي مسافة بين الجذر و الطرف العلوي للريشة & $\mathrm{Z}$ \\
\hline$m^{2}$ & مساحة مقطع الريشة عند أي نقطة على طول الريشة & $\mathrm{Az}$ \\
\hline $\mathrm{m} / \mathrm{s}$ & سر عة دور ان الريشةة & $V_{\mathrm{b}}$ \\
\hline $\mathrm{m}$ & معدل المسافة من مركز عمود الدور ان الى الحافة الأمامية للريشة & $r_{\mathrm{ave}}$ \\
\hline $\mathrm{m} / \mathrm{s}$ & السرعة المطلقة للبخار المغادر الخانق & $v s_{1}$ \\
\hline $\mathrm{m} / \mathrm{s}$ & السرعة المطلقة للبخار المغادر للريشة & $\mathrm{VS}_{2}$ \\
\hline $\mathrm{m} / \mathrm{s}$ & السر عة النسبية للبخار الداخل للريشة & $v r_{1}$ \\
\hline $\mathrm{m} / \mathrm{s}$ & السر عة النسبية للبخار المغادر للريشة & $v r_{2}$ \\
\hline- & معامل سرعة الريشة & $K_{v}$ \\
\hline Ton/hr. & معدل تدفق البخار & $m^{0}$ \\
\hline $\mathrm{m} / \mathrm{s}$ & محصلة سر عة الطاقة & Vw \\
\hline $\mathrm{m} / \mathrm{s}$ & المركبة الاولى لمحصلة سر عة الطاقة & $\mathrm{vw}_{1}$ \\
\hline $\mathrm{m} / \mathrm{s}$ & المركبة الثانية لمحصلة سر عة الطاقة & $\mathrm{vw}_{2}$ \\
\hline $\mathrm{N}$ & القوة الناتجة عن البخار & $F_{s t}$ \\
\hline $\mathrm{N}$ & القوة المحورية & $F_{a x}$ \\
\hline$m^{2}$ & المساحة السطحية التي يضرب بها البخار & $A_{S}$ \\
\hline $\mathrm{N} / m^{2}$ & الضغط الناتج عن البخار & $p$ \\
\hline $\mathrm{m}$ & كتلة العمود الر ابط & $m^{\prime \prime}$ \\
\hline
\end{tabular}

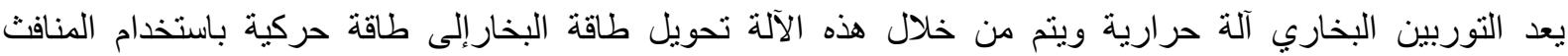
ومن ثم تحويل الطاقة الحركية إلى قوة تتجز "شغل ميكانيكي" باستخدام الريش ـ تصنف التئ التوربينات البخارية على نو عين أساسيين هما: 1. التوربين الدفعي(Impulse turbine) 2. توربين ردالفعل (Reaction turbine).

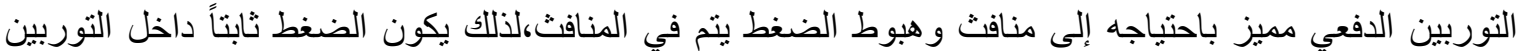

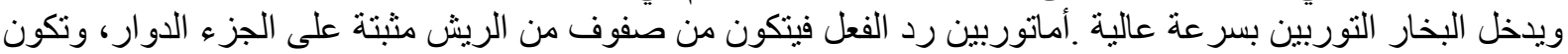

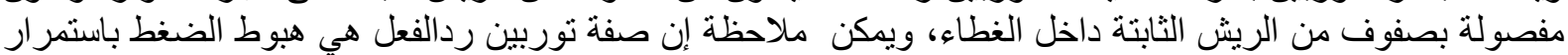

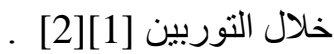

\section{2- التوربين في محطة بيجي}

ويتكون التوربين من مقطعين [3] :-

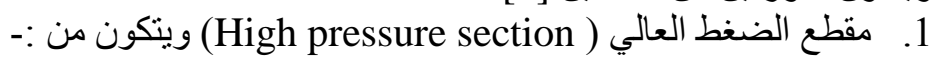
أـ مرحلة الضغط الضعالي م (High pressure stage) ويتكون من تسع مر احل من الريش.

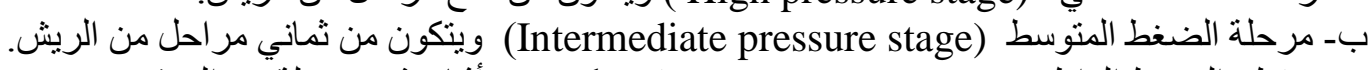

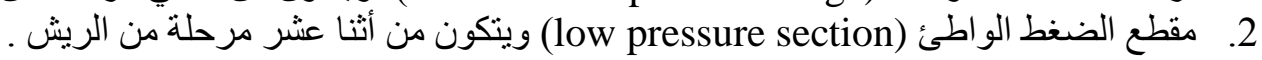




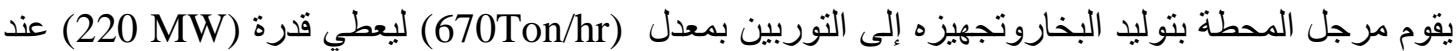

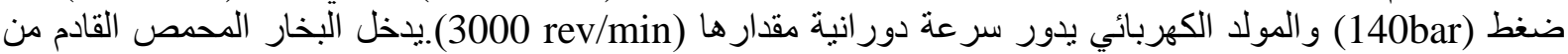

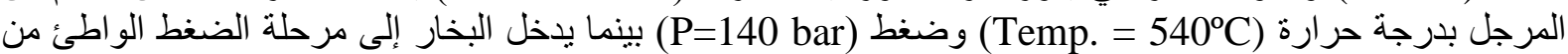

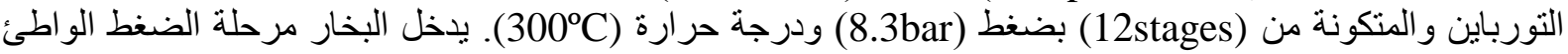

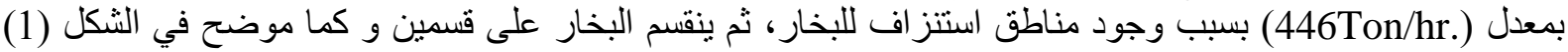

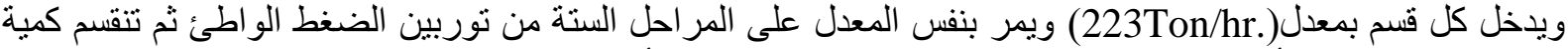

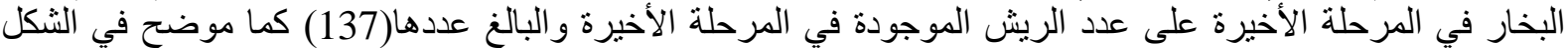

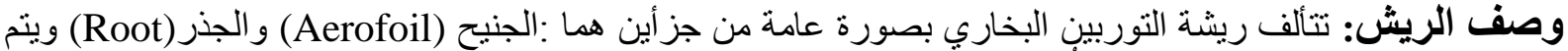

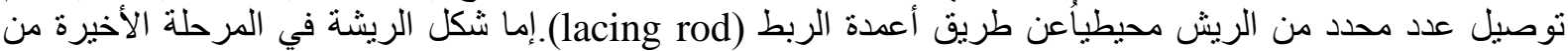

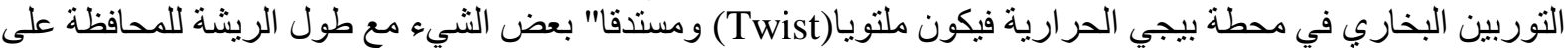

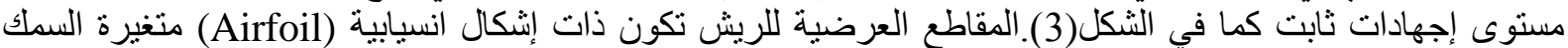

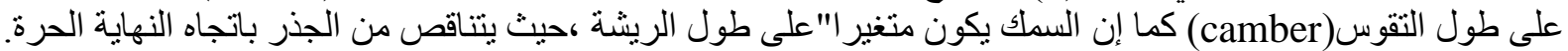

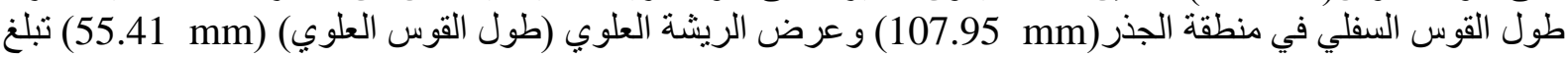
زاوية الالتواء للريشة في هذه المرحلة (43 degree)عرض الريشة قبل الالتواء (72.2 mm) سمك الريشة قبل

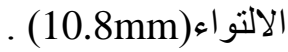

$$
\text { وفي المر احل الأخيرة من الريش يستعاض عن طوق التقوية (Shroud) بأعمدة ربط : }
$$

1. عمود ربط داخلي صلد يبلغ قطره (7.4mm) ويقع على بعد(10 mm (360) من قاعدة الريشة (الجذر).

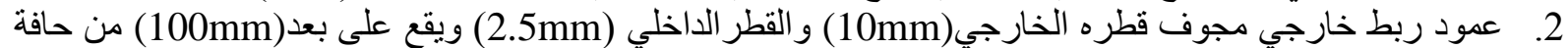

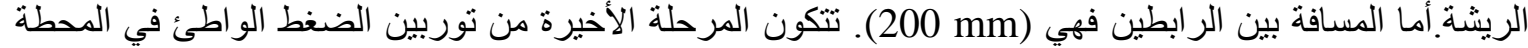

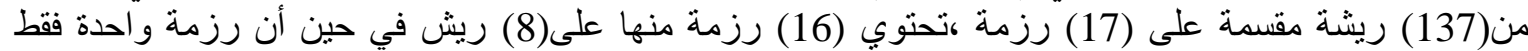

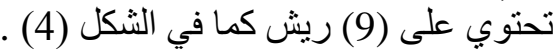
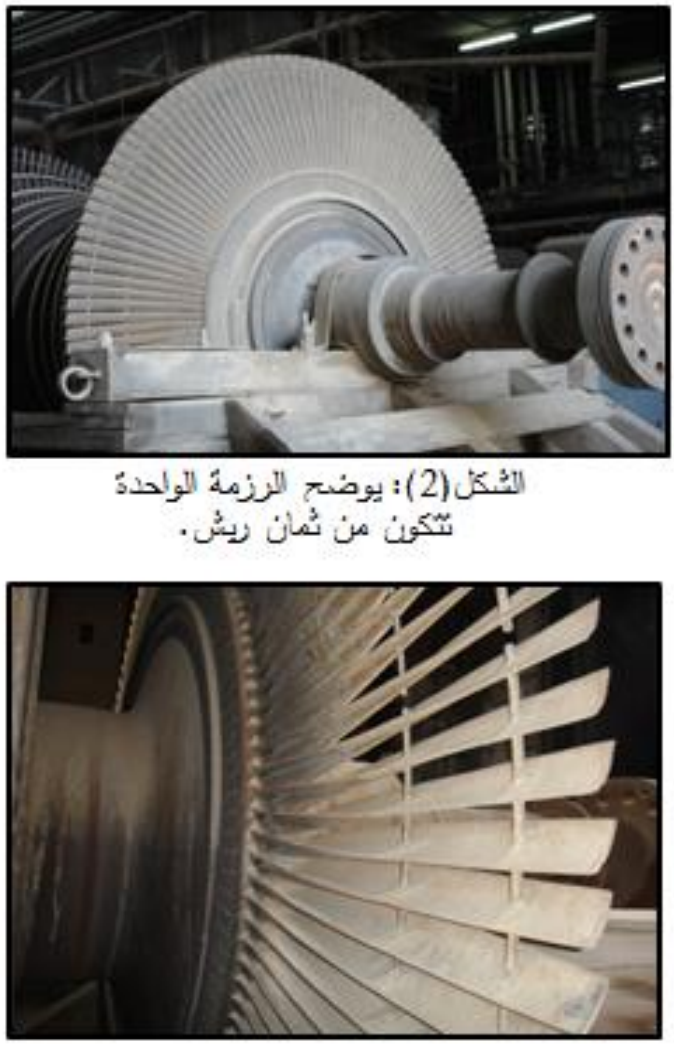

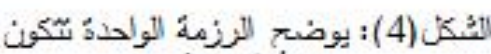

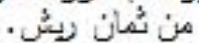

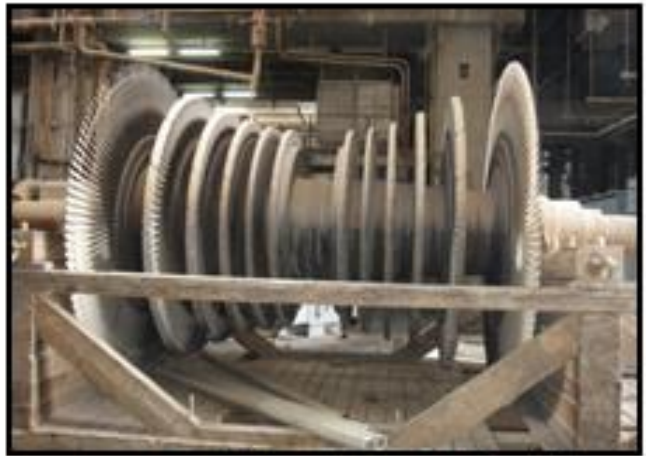

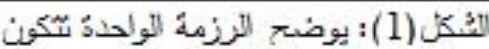

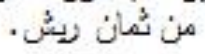

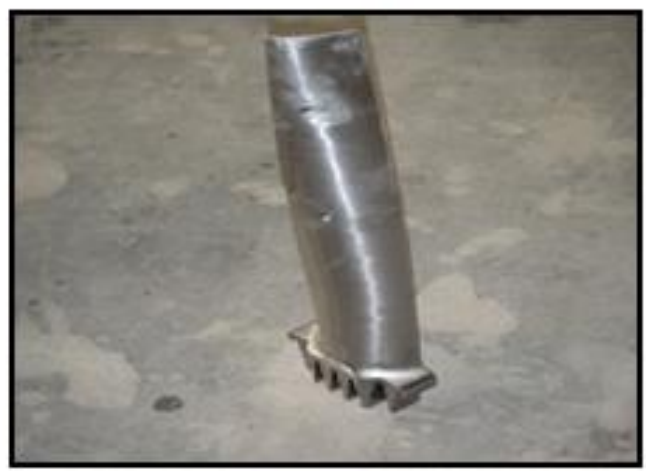

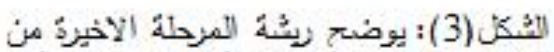

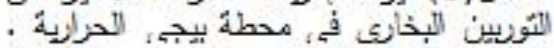


وقد حدد الباحثان (Mayer\&Besigk) (4) ثلاثة عوامل تحدد قيمة الاجهادات هي حسابات التصميم،عمليات

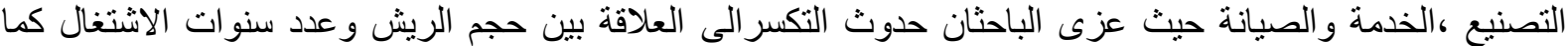

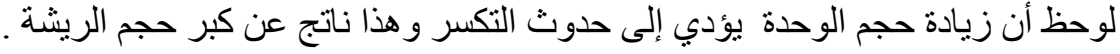

وقام الباحث(الجبوري،عمر خليل) 2001 [5] دراسة العوامل التي أدت إلى كسر ريش المراحل الأخيرة لتوربينات

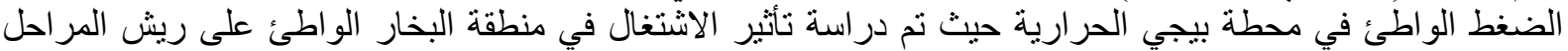

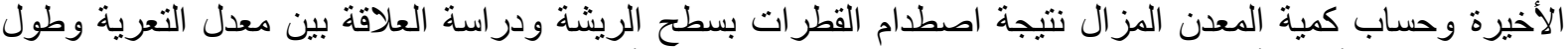

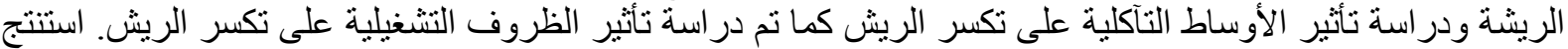

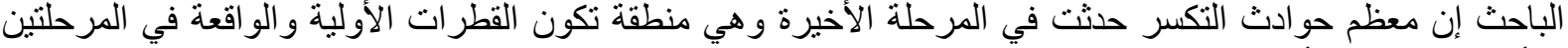

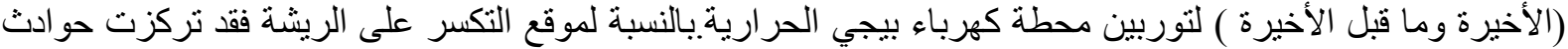

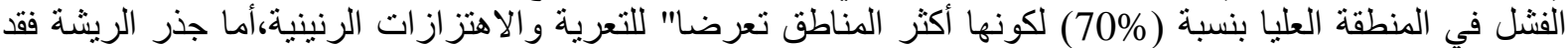

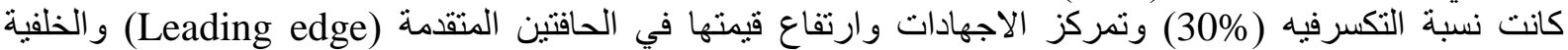

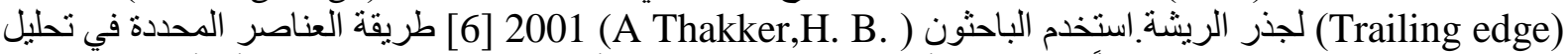

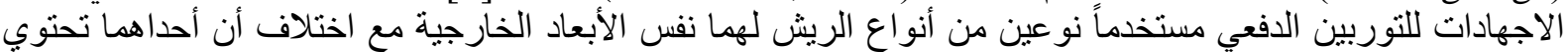

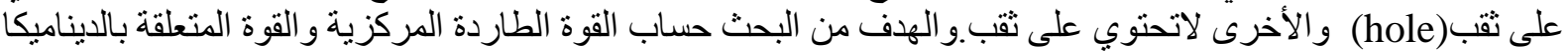

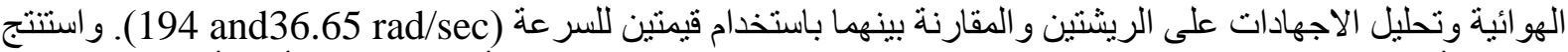

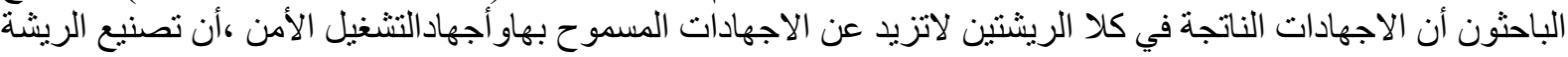

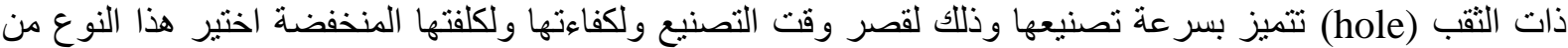

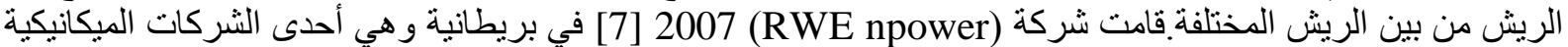

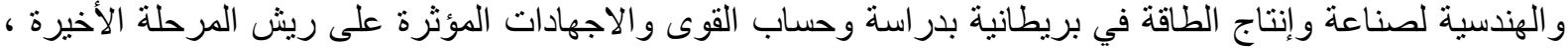

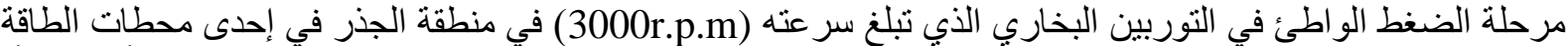

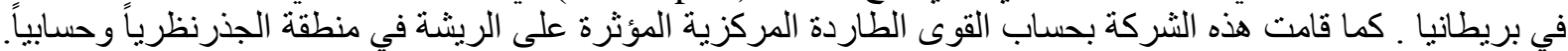

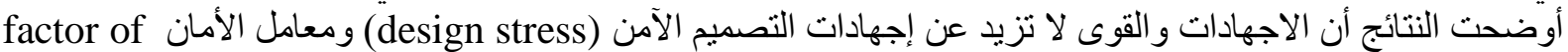

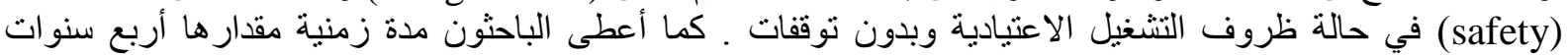
للفحص الدوري للريش و لاسيما في هذه المرحلة وذللك للكثف المبكر تبن عن بداية الثنقوق وعن تكسر الريش.

قام الباحث (8rkan K. Husain Al-Taie) 2008) 20دراسة إحدى الطرق لتقليل القوة الطاردة المركزية وذللك

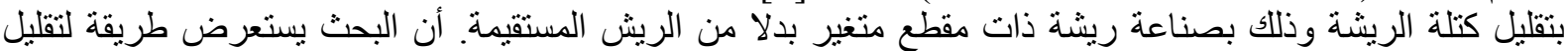
مساحة مقطع الريشة وكذلك تم استعر اض تحليل لهذه الريشة ـ و وقام الباحث بدر اسة القوة الطاردة الرئة المركزية مستخدما ثلاثة

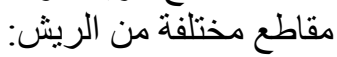

1. مساحة مقطع الريشة ثابت على طول الريشة (constant cross-sectional area). 2. مساحة مقطع الريشة متغير على طول الريشة (variable cross-sectional area). 3. مساحة مقطع الريشة متغير على طول الريشة وأن مساحة مقطع الريشة أقل مما في الحالة B.

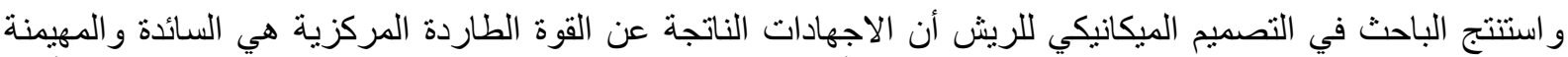

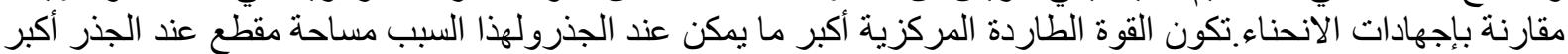

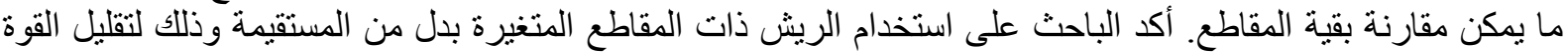

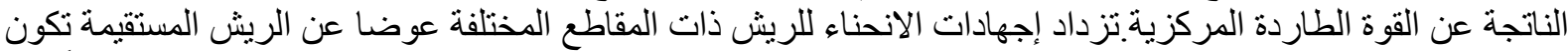

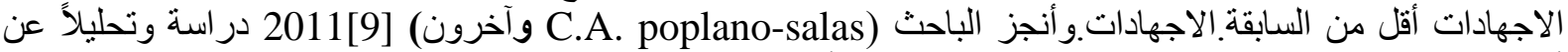

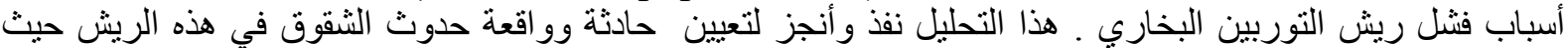
أكتثف ولوحظ أنه حصلت ونشأت هذه الثقوق في المنطقة المقواة (reinforcing)، عند أعمدة الربط في الجزء العهاء العلوي

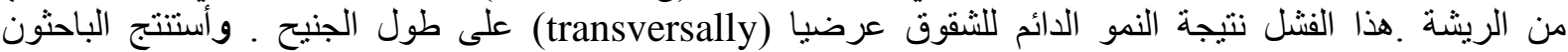

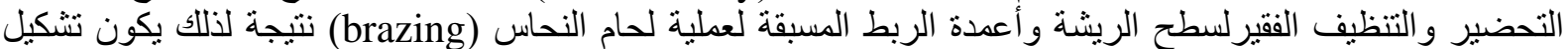

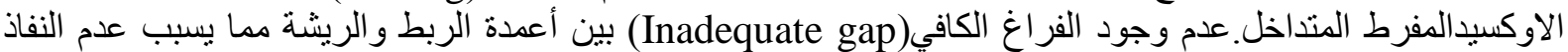

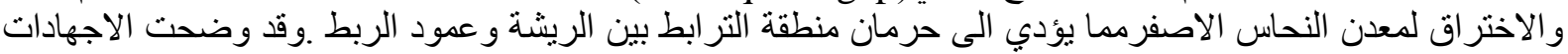

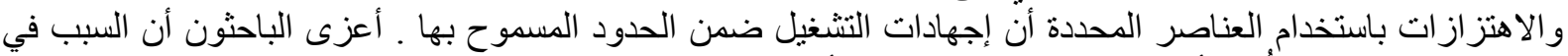

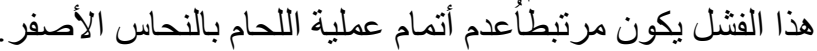




\section{3- الأسساس النظري \\ الاجهادات المؤثرةعلى ريش التوربين البخاري: \\ هناك نوعان من الاجهادات المؤثرة على ريش التوربين البخاري هي [2]:

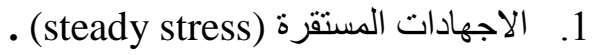 \\ 2. الاجهادات المتغيرة (alternating or vibratory stress) .}

الاجهادات المستقرة المؤثرة على ريش التوربين البخاري الناتجة عن مجمو عة من الأحمال بسبب القوة الطاردة المركزية

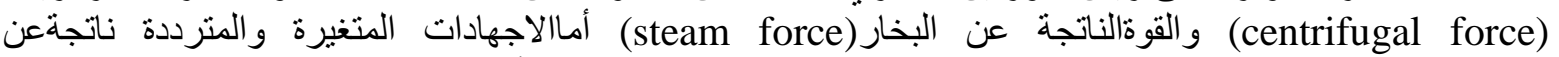
تغيرواضطر اب جريان البخار ، وظاهرة الرنين .وتعدالاجهادات المستقرة الأكثر دقة في الحسابات من الاجهادات

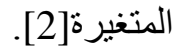

\section{4-القوى المؤثرةعلى ريشة التوربين البخاري \\ 1 1. القوةالطاردة المركزيةالناتجة عن سر عة الدور ان العالية .

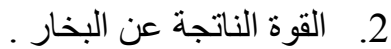

\section{5-حساب القوة الطاردة المركزية المؤثزة على الريشة}

الثكل (5) يوضح أبعاد ريشة المرحلة الأخيرة من التوربين البخاري في محطة بيجي الحرارية يمكن حساب القوة الطاردة المركزية من المعادلة العامة لحساب القوة الطاردة المركية المركزية الآتية:

$\mathrm{F}_{\mathrm{cf}}=\mathrm{mr} \omega^{2}$

حيث تتناسب القوة الطاردة المركزية طرديا مع كتلة الريش وكثافة معدن الريشة وطول الريشة ومساحة مقطع الريشة و

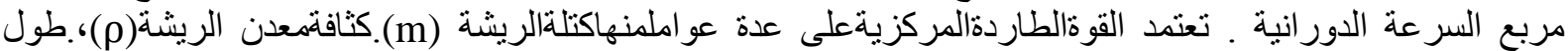

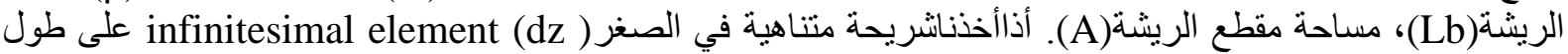

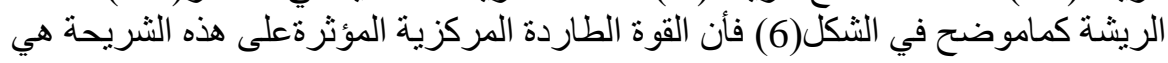

$\mathrm{df}_{\mathrm{cf}}=\mathrm{dm} \cdot \omega^{2}\left(\mathrm{R}_{\mathrm{r}}+\mathrm{z}\right)$

Where :

$\mathrm{dm}=\rho \cdot \mathrm{A}(\mathrm{z}) \mathrm{dz}$

$\mathrm{df}_{\mathrm{cf}}=\rho \cdot \omega^{2} \cdot A(\mathrm{z}) \cdot\left(\mathrm{R}_{\mathrm{r}}+\mathrm{Z}\right) \mathrm{dz}$

$\mathrm{F}_{\mathrm{cf}}(\mathrm{x})=\int_{\mathrm{x}}^{\mathrm{lb}} \rho \cdot \omega^{2} \cdot \mathrm{A}(\mathrm{z}) \cdot\left(\mathrm{R}_{\mathrm{r}}+\mathrm{Z}\right) \mathrm{dz}$

مكن اعتبار الريشة (Cantilever with variable cross-section area) عنلةجاسئة متغيرة المقطع مثبتة من الجذروان مساحة مقطع الريشة تتغير وفق المعادلة الآتية[8]:

$\left(\frac{\mathrm{A}(\mathrm{z})}{\mathrm{Ar}}\right)^{\mathrm{lb}}=\left(\frac{\mathrm{At}}{\mathrm{Ar}}\right)^{\mathrm{z}}$

$\frac{\mathrm{A}(\mathrm{z})}{\mathrm{Ar}}=\left(\frac{\mathrm{At}}{\mathrm{Ar}}\right)^{\mathrm{z} / \mathrm{lb}}$

$\mathrm{A}(\mathrm{z})=\operatorname{Ar} \cdot\left(\frac{\mathrm{At}}{\mathrm{Ar}}\right)^{\mathrm{z} / \mathrm{lb}}$ 
$\mathrm{F}_{\mathrm{cf}}=\rho \cdot \omega^{2} \int_{\mathrm{x}}^{\mathrm{lb}}\left[\operatorname{Ar} \cdot\left(\frac{\mathrm{At}}{\mathrm{Ar}}\right)^{\frac{\mathrm{z}}{\mathrm{lb}}}\right] \cdot\left(\mathrm{R}_{\mathrm{r}}+\mathrm{Z}\right) \mathrm{dz}$

$\mathrm{F}_{\mathrm{cf}}=\rho \cdot \omega^{2} \operatorname{Ar}\left[\mathrm{R}_{\mathrm{r}} \int_{\mathrm{x}}^{\mathrm{lb}} \operatorname{Ar} \cdot\left(\frac{\mathrm{At}}{\mathrm{Ar}}\right)^{\frac{\mathrm{z}}{\mathrm{lb}}} \cdot d z+\int_{\mathrm{x}}^{\mathrm{lb}} \operatorname{Ar} \cdot \mathrm{z}\left(\frac{\mathrm{At}}{\mathrm{Ar}}\right)^{\frac{\mathrm{z}}{\mathrm{lb}}} \cdot \mathrm{dz}\right]$

بعد حل وإيجاد التكامل الموجود في المعادلةرقم (10) نحصل على قيمة القوة الطاردةالمركزية في أي نقطةعلى طول الريشة كما في المعادلة الآتية :

$\mathrm{F}_{\mathrm{cf}}(\mathrm{x})=\rho \cdot \omega^{2}\left[\frac{\operatorname{Ar} \cdot\left(\frac{\mathrm{At}}{\mathrm{Ar}}\right)^{\frac{\mathrm{z}}{\mathrm{lb}}} \cdot \operatorname{Rr} \cdot \mathrm{lb}}{\ln \left(\frac{\mathrm{At}}{\mathrm{Ar}}\right)}+\frac{\operatorname{Ar} \cdot\left(\frac{\mathrm{At}}{\mathrm{Ar}}\right)^{\frac{\mathrm{z}}{\mathrm{Ib}}} \cdot \mathrm{z} \cdot \mathrm{lb}}{\ln \left(\frac{\mathrm{At}}{\mathrm{Ar}}\right)}-\frac{\operatorname{Ar} \cdot\left(\frac{\mathrm{At}}{\mathrm{Ar}}\right)^{\frac{\mathrm{z}}{\mathrm{lb}}} \cdot \mathrm{lb}^{2}}{\left[\ln \left(\frac{\mathrm{At}}{\mathrm{Ar}}\right)\right]^{2}}\right]_{\mathrm{x}}^{\mathrm{lb}}$

6-نموذج من الحسابات : - 20 -

من المعادلة (11) بمكن حساب القوة الطاردة المركزية على أي نقطة على طول الريشة وذللك بالتعويض بالقيم

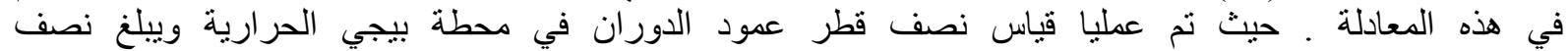
قطر عمودالدور ان (Rr= 822 mm) وطول الريشة الكلي (Lb =660 mm) ومن أمكانبة وتقنية برنامج(الأوتوكاد)تم

حساب مساحة مقطع الريشة العلوي(At=150.95mm) مساحة مقطع الريشة عند الجذر (Ar=1490.195mm)

إذا عوضناعن قيمة (x) وهي أي نقطة على طول الريثة وعن بقية القيم في معادلة رقم (11) نحصل على الرئل

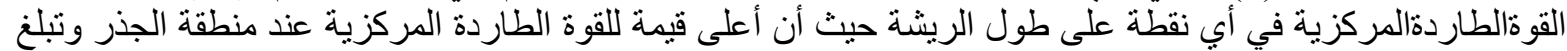

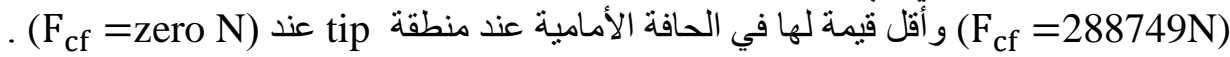

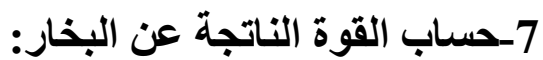

من الثكلان(7)، (8) - مكن حساب القوة الناتجة عن البخار

$\mathrm{V}_{\mathrm{b}}=\omega \times \mathrm{r}_{\mathrm{ave}}$

$\mathrm{r}_{\mathrm{ave}}=\frac{660+822}{2}=0.741 \mathrm{~mm}$

$\mathrm{V}_{\mathrm{b}}=314 \times 0.741=233 \mathrm{~m} / \mathrm{s}$

من المعلومات العملية التي حصلنا عليها من محطة بيجي الحرارية ومن الدراسات والبحوث التي أجراها

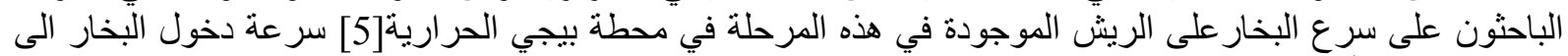

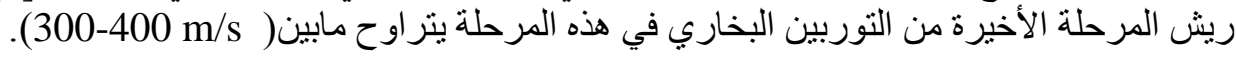
نفرض أن سر عة دخول البخار إلى المرحلة الأخيرة من توربين الضغط الواطئ في المحطة تبلغ ( m/s 400). $\mathrm{vs}_{1}=400 \mathrm{~m} / \mathrm{s}$

من مر اجعة مخططات الثركة الايطالية المصنعة للتوربين البخاري في بيجي أن زاوية المنفت (Nozzle): $\alpha_{1}=22^{\circ}$

تطبيق قانون الجيب التمام على مثلث السرعةالموضح في الثكل (8)

$\mathrm{vr}_{1}^{2}=\mathrm{vs}_{1}^{2}+\mathrm{vb}^{2}-2 \mathrm{vs}_{1} \cdot \mathrm{vbcos}_{1}$

$\mathrm{vr}_{1}{ }^{2}=400^{2}+233^{2}-2 \times 400 \times 233 \times \cos (22)$

$\mathrm{vr}_{1}=204 \mathrm{~m} / \mathrm{s}$ 
من التماتل (Symmetrical) الموضح في الثكل(8)

$\beta_{1}=\beta_{2}$

إذا اعتبرنا تأثير احتكالك البخار بالريشة مساويا الى الصفر

$\mathrm{K}_{\mathrm{v}}=\frac{\mathrm{vr}_{2}}{\mathrm{vr}_{1}}$

$\because \mathrm{vr}_{1}=\mathrm{vr}_{2}$

(Blade velocity coefficient $\mathrm{K}_{\mathrm{v}}=1$ )

$\mathrm{K}_{\mathrm{v}}<1$

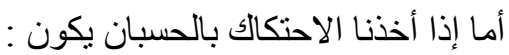

بتطبيق قانون الجيب (the sine rule in triangle)على مثلث السرعة الموضح في الثكل(8)

$\frac{\mathrm{vr}_{1}}{\sin \alpha_{1}}=\frac{\mathrm{vs}_{1}}{\sin \beta_{1}}$

$$
\frac{204}{\sin 22}=\frac{400}{\sin \beta_{1}}
$$

$\sin \beta_{1}=0.7345$

$\beta_{1}=47.265^{0}$

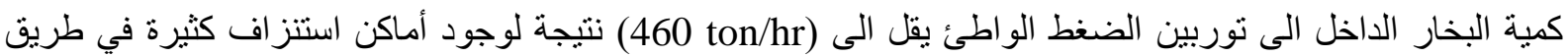

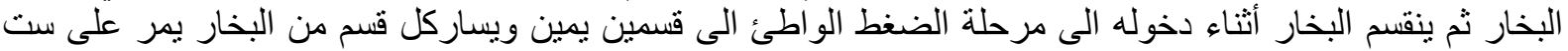
مر احل وفي المرحلة الأخيرة ينقسم البخار الى عدد الريش المئ الموجودة في هذه المرحلة (137) ريشة .

كمية البخار الاخل الى كل ريشة

من العزم الدفعي (impulse momentum) يمكن حساب القوة الناتجة عن البخار على كل ريشة [10,11].حيث تعتمد القوة الناتجة عن البخار على معدل تغير الزخم

Force $=\mathrm{m}^{0} \times$ change in velocity

كمية البخار الداخل الى كل جهة من مرحلة الضغط الواطئ حيث ينقسم البخار الى قسمين يمين ويسار $\mathrm{m}^{0}=\frac{446}{2}=223 \mathrm{Ton} / \mathrm{hr}$.

كمية البخار الداخل الى كلريشة من ريشالمرحلة الأخيرة من توربين الضغط الواطئ حيث ينقسم البخار الى (137) ريشة $\mathrm{m}^{0}=\frac{223 \times 1000}{3600 \times 137}=0.452 \mathrm{~kg} / \mathrm{sec}$

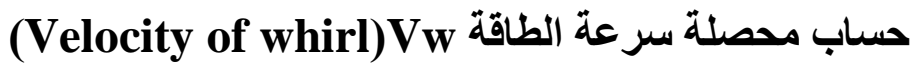

من مثلث السرعة (velocity diagram) الموضح في الثكل (8) يمكن حساب محصلة سر عة الطاقة و التي تكون بأتجاه

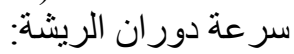

$\mathrm{Vw}=\mathrm{vw}_{1}+\mathrm{vw}_{2}$

$\mathrm{Vw}=\mathrm{vr}_{1} \cos \beta_{1}+\mathrm{vr}_{2} \cos \beta_{2}$ )

أما القوة الناتجة عن البخار فيمكن حسابها من المعادلة الاتية :

$\mathrm{F}_{\mathrm{st}}=\mathrm{m}^{0}\left(\mathrm{vw}_{1}+\mathrm{vw}_{2}\right)$

$\mathrm{F}_{\mathrm{st}}=\mathrm{m}^{0}\left(\mathrm{vr}_{1} \cos \beta_{1}+\mathrm{vr}_{2} \cos \beta_{2}\right)$ 
$\mathrm{F}_{\mathrm{st}}=0.452(204) \cos 47.265+204 \cos 47.625$

$\mathrm{F}_{\text {st }}=0.452 \times(276.87)=125 \mathrm{~N}$

$\mathrm{F}_{\mathrm{ax}}=\mathrm{m}^{0}\left(\mathrm{vr}_{1} \sin \beta_{1}-\mathrm{vr}_{2} \sin \beta_{2}\right)$

و القوة المحورية (axial thrust) تحسب في المعادلة الاتية:

تهمل هذه القوة في حالة التوربين الدفعي لأنها قليلة ويمكن اعتبار ها مساوية الى الصفر .

حساب المساحة السطحية الناتجة عن ضغط البخار

أذا فرضنا أن المساحة التي يضرب بها البخار الريشة عبارة عن شبه منحرف ويمكن حساب مساحة شبه المنحرف من

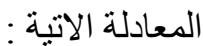

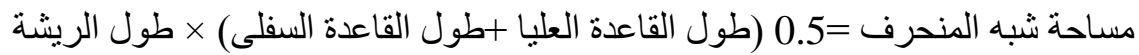

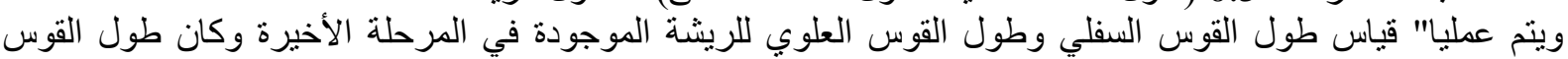

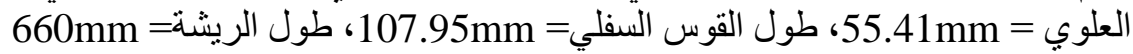
المساحة التي يضرب بها البخار الريشة :

$\mathrm{A}_{\mathrm{s}}=53908.8 \mathrm{~mm}^{2}$

$\mathrm{A}_{\mathrm{s}}=53908.8 \times 10^{-6} \mathrm{~m}^{2}$

$p=\frac{F_{s t}}{A_{s}}$

وينم حساب الضغط الناتج عن قوة البخار نطبق المعادلة التالية : p $=2318.7 \mathrm{~N} / \mathrm{m}^{2}$

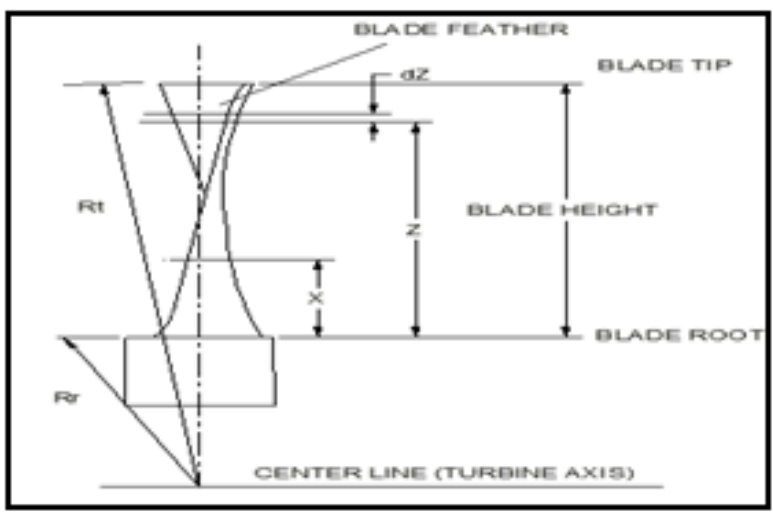

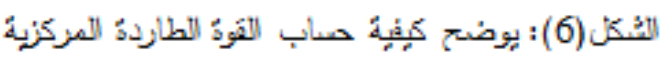

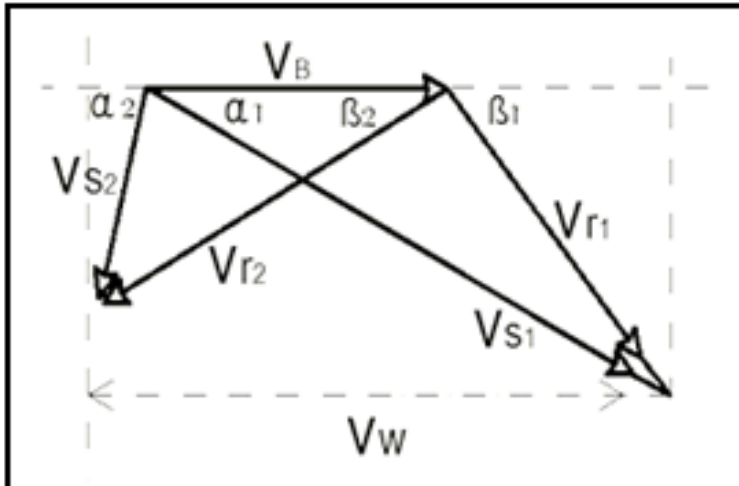

الشُكل (8) : يوضح زوليا وسزع نخول

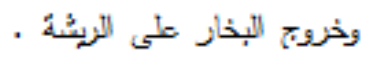

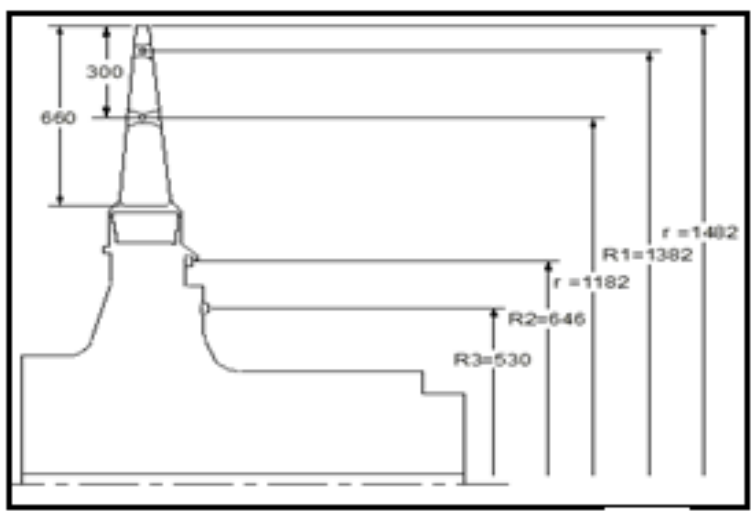

الإنكل (5): يوضح لبعاد الزيشة.

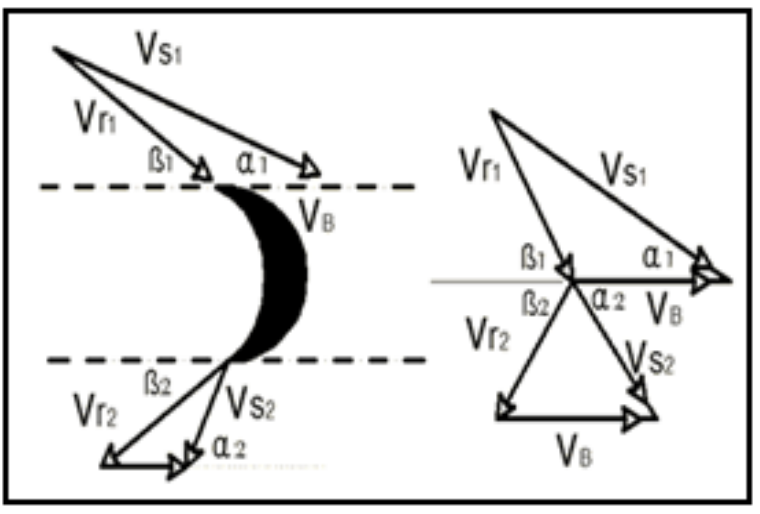

الاُكل (7) : يوضح نزوليا وسزع نخول

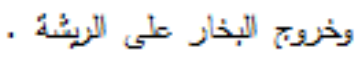




\section{تقتيات البرامج المستخدمة في رسم وأيجاد قيم الاجهادات على الريشة}

نظر ألشكل الثلاثي الابعاد المعقد للريشة وتغير مساحة الريشة على طول الريشة وتغير السمك على طول الريشة

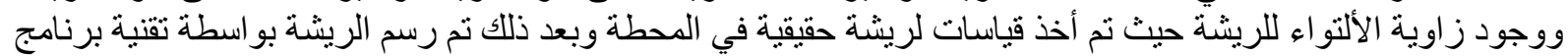

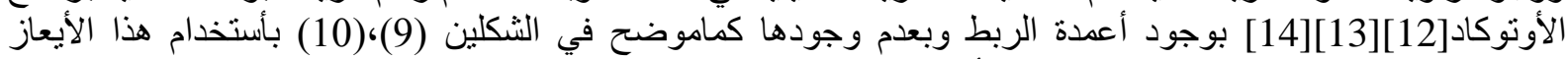

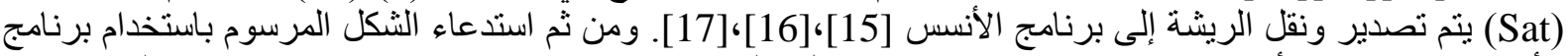

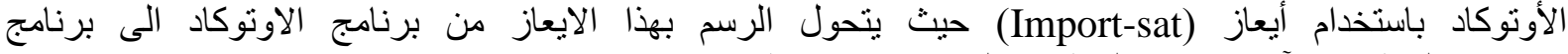

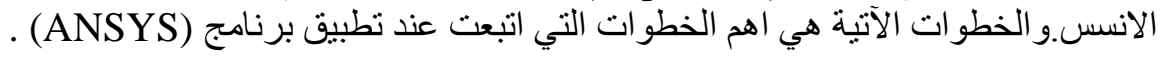

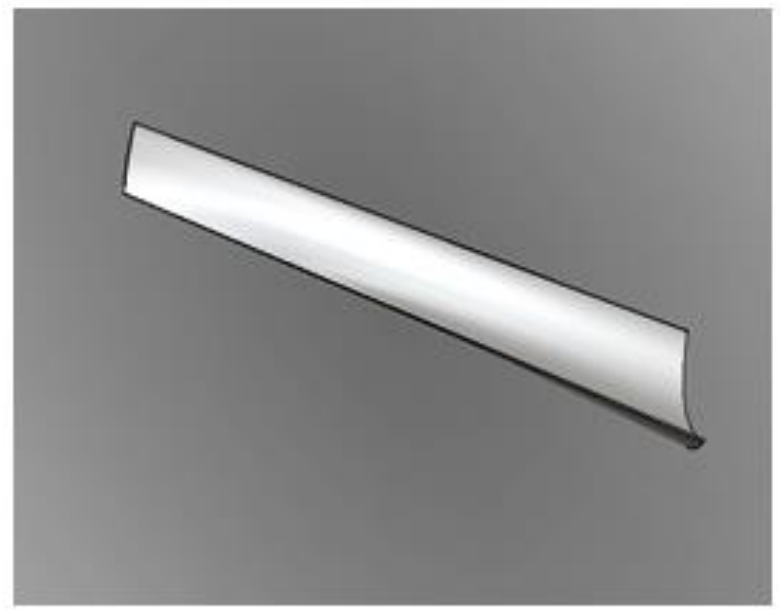

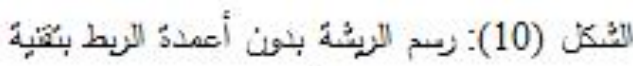

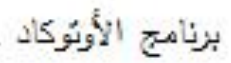

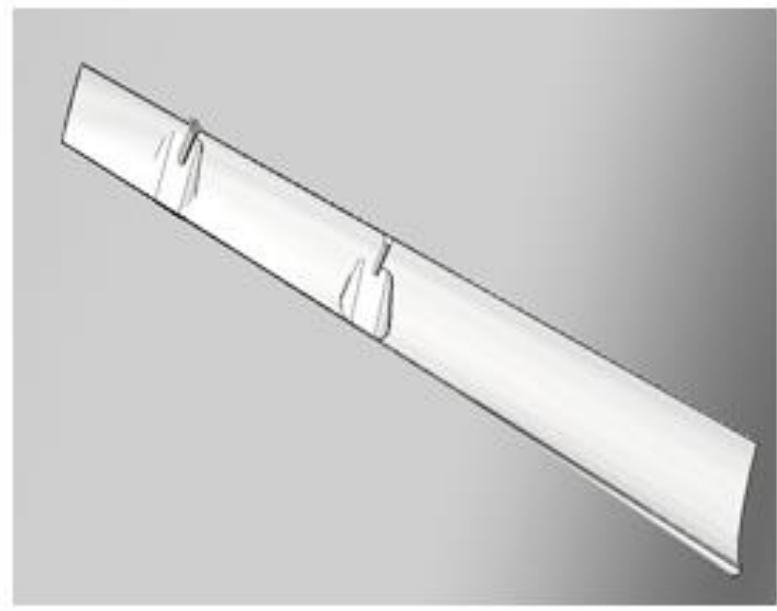

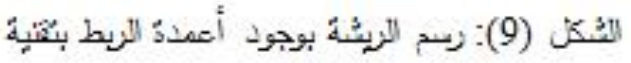

$$
\begin{aligned}
& \text { برناهِ الأوبُوكاد. }
\end{aligned}
$$

preprocessor (I)

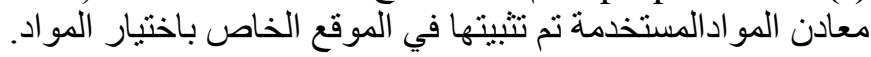

1- اضيفت الخواص الميكانيكية الخاصة بالمعدن المستخدم في بناء النموذج، مثل معامل المرونة ، الكثافة

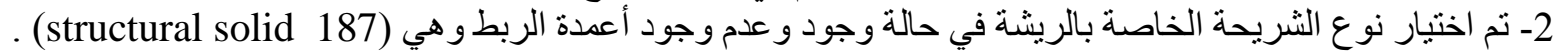
3- قسم النموذج الي الشريحة المختارة في اعلاه في حالة وجود عمود الربط ودي وفي حالة عدم وجوده وكما مبين في الثكل

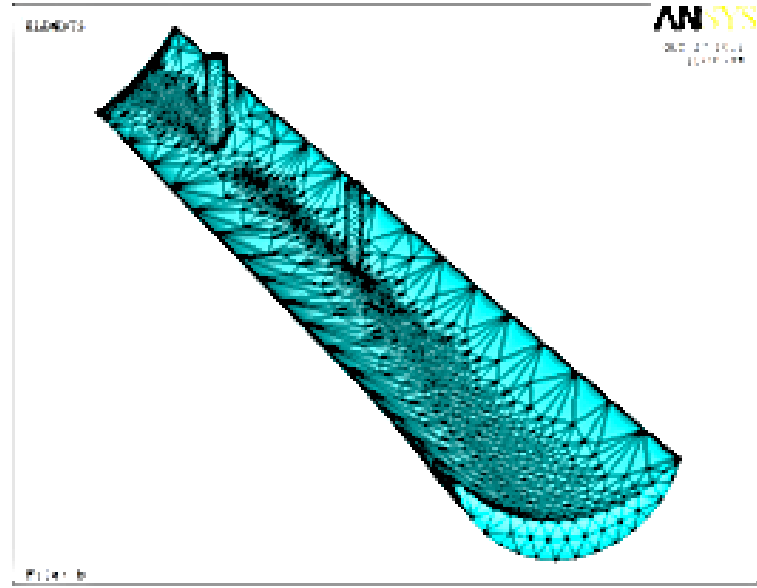

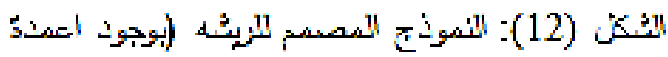

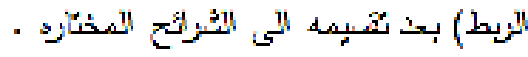

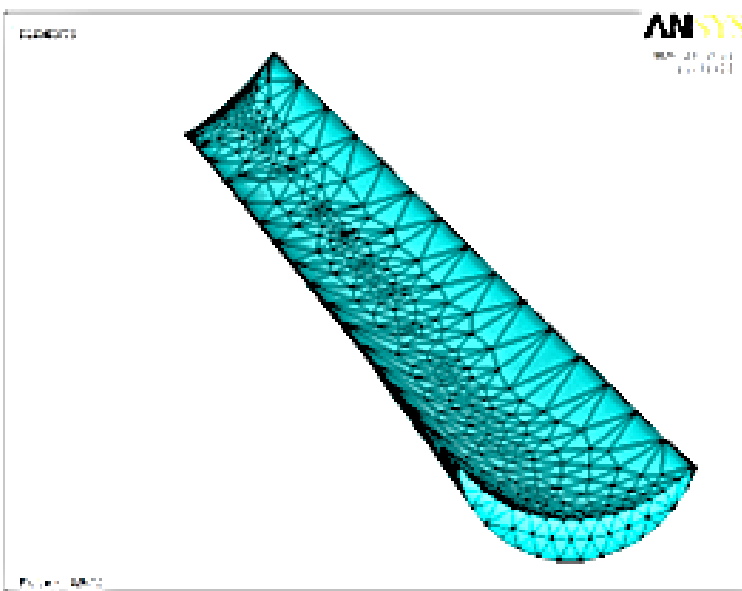

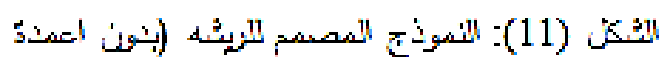

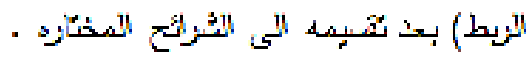


solution (II)

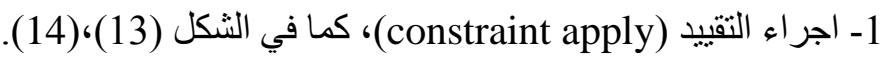

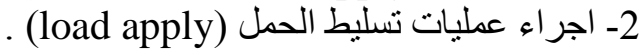
3- اجراء عمليات تنظيم عدد ال (load step) (lub steps)، وكذاللك الـ (lop).

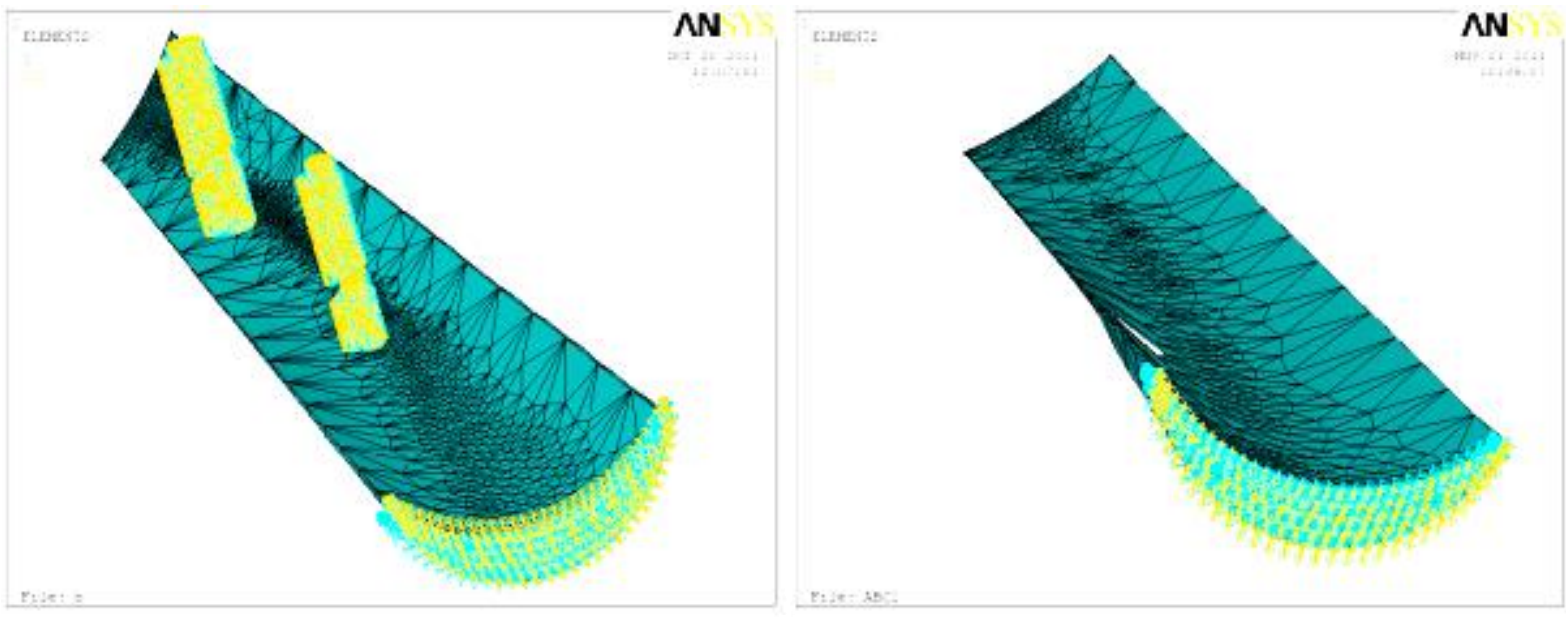

$$
\begin{aligned}
& \text { الثكل (14) : النموذج المصمم للريشه (بدون وجود اعمدة الشكل (13): النموذج المصمم للريشه (بوجود اعمدة الربط) } \\
& \text { بعد تقسيمه الى الشرائح المختاره . } \\
& \text { الربط) بعد تقسيمه الى الثرائح المختاره . }
\end{aligned}
$$

يكن post processor (III)

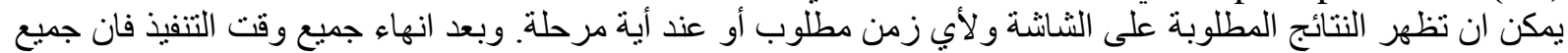

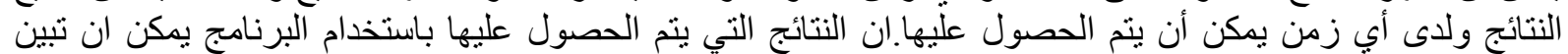

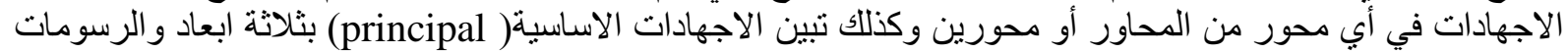

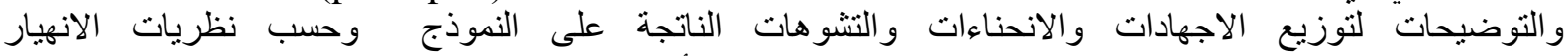
المرن.و الفرضيات المتبعة في بناء نموذج الريشة و وعلية ايجاد قيم الأجهادات على التهات الريشة :

1. الريشة مقيدة الجذر في جميع الاتجاهات (X,Y,Z) وتم لحظيا"تدوير الريشة بأتجاه المحور(X) وتحليل الاجهادات المستقرة عليها.

2. الريثة مقيدة الجذر ومقيدة أعمدة الربط في حالة وجودها في جميع الاتجاهات (X,Y,Z) وتم لحظيا"اتدوير الريشة

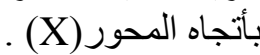

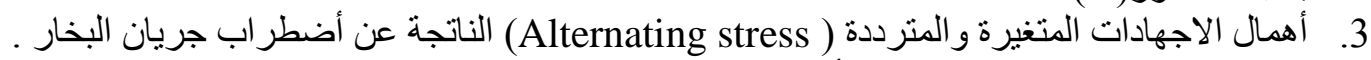

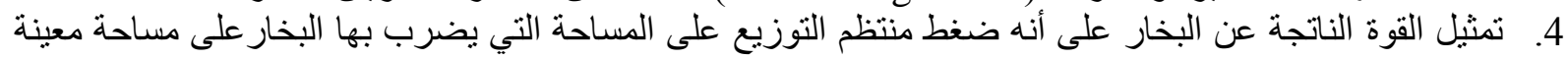
من الريشة . منئة

\section{:تنائج برنامج الانسس :}

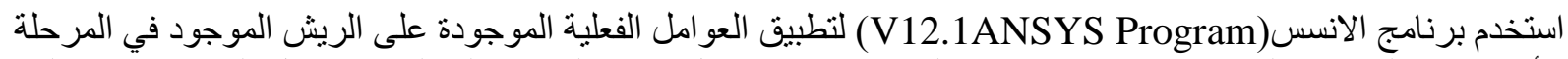

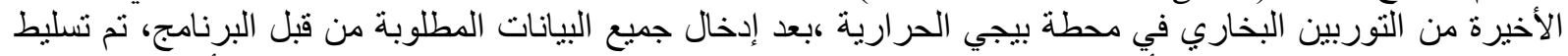

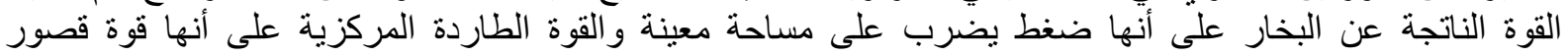

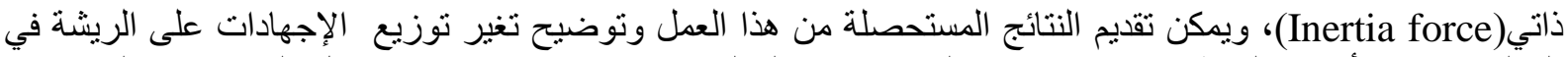
الحالتين وجود أعمدة الربط وعدم وجودها والمقارنة بين الحالتين برسومات ومناقتنتها بتنتاول المنحنيات و الرسومات الريت

$$
\text { المعروضة على النحو الأتي: }
$$

أعدة الثكل (15): يوضح الأجهاد المكافئ (أجهادات فون ميسس) الناتج عن القوة الطاردة المركزية في حالة عدم وجوداد

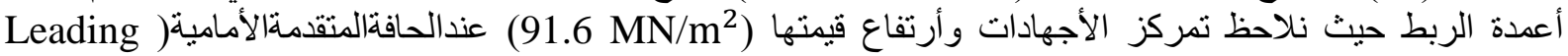


(Trailing edge) لجذر الريشة وتقل الأجهادات كلماتجهنا باتجاه الطرف العلوي للريشة وتكون أقل

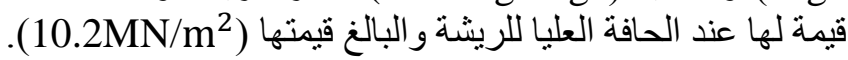

• الثكل (16): يوضح (أجهادات فون ميس) الناتج عن قوة البخار حالة عدم وجود أعمدة الربط حيث نلاحظ تمركز

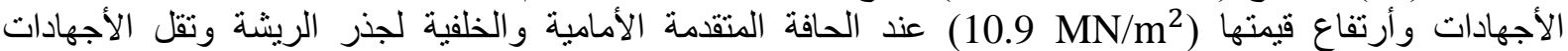

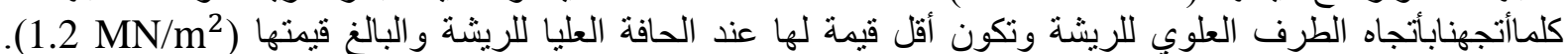

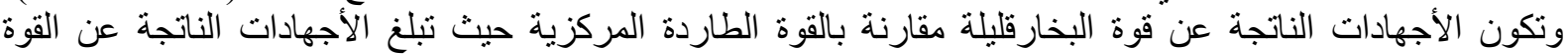

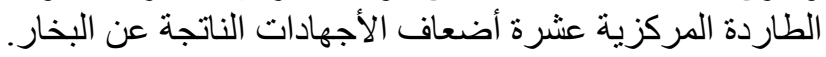

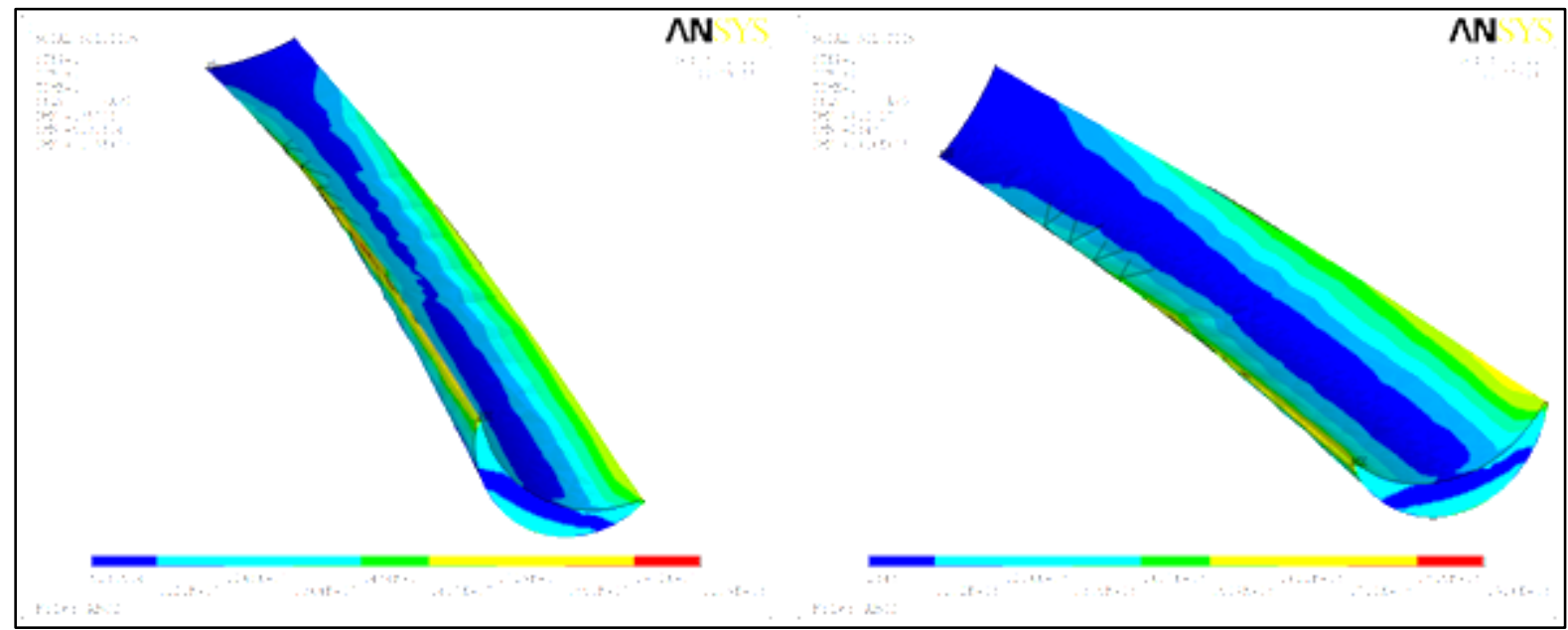

الثكل (16) : الاجهاد المكافيء الناتج عن قوة

الثكل (15) : الاجهاد المكافيء الناتج عن القوة البخار فى حالة عدم وجود أعمدة الربط .

الطاردة المركزية في حالة عدم وجود أعمدة الربط .

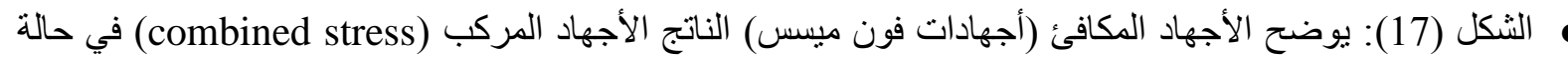

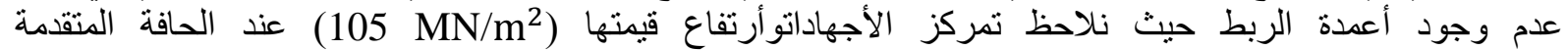

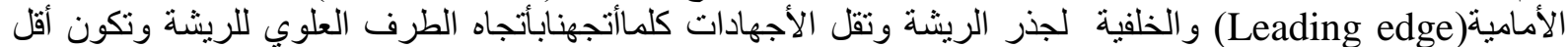

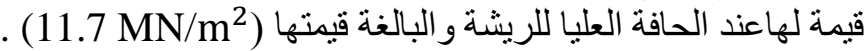

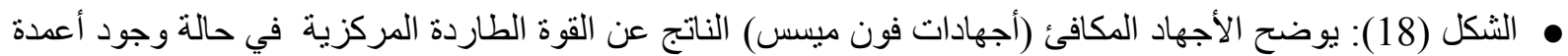
الربطحيث نلاحظ تمركز الأجهاداتوأرتفاع قيمتها (76MN/m²) حول العمود الرابط الصلافي الصافي منطقة الألتحام بالريشة

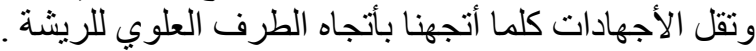

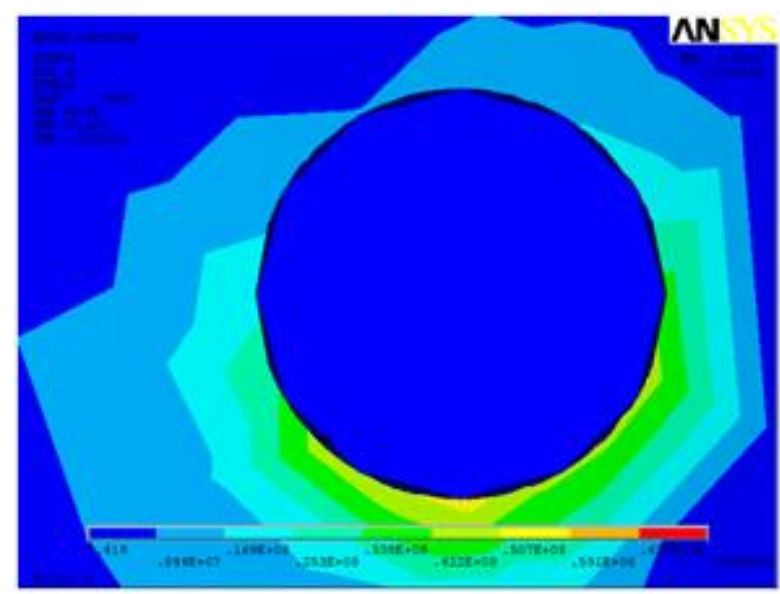

الثكل (18) : الاجهاد الناتج عن القوة الطاردة

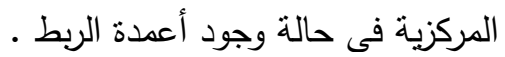

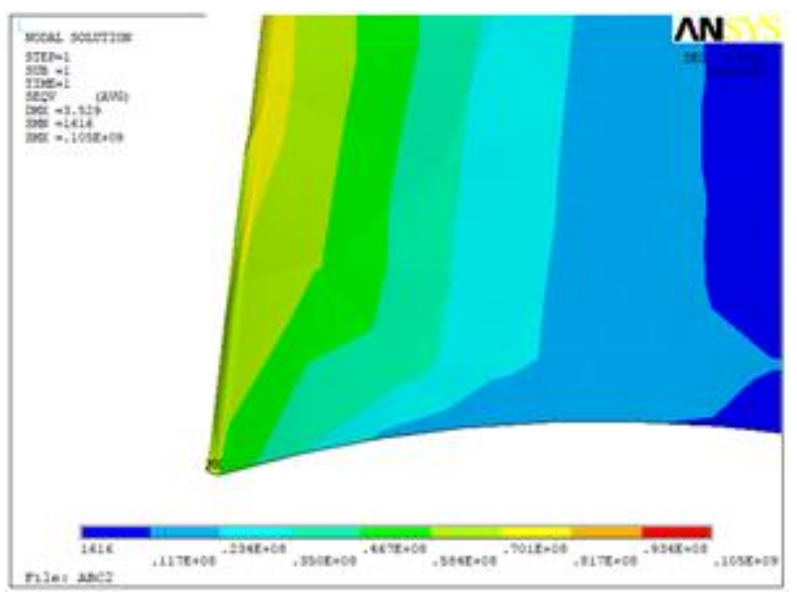

الثكل (17) : تمركز الاجهاد المكافيء الناتج عن الأجهاد

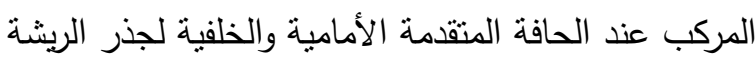




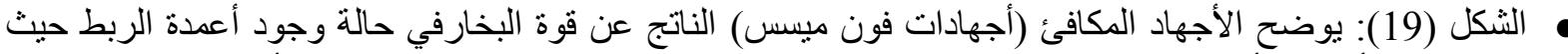

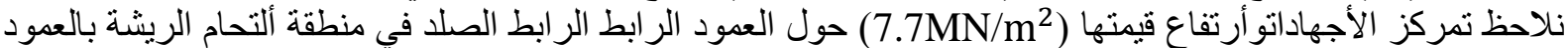

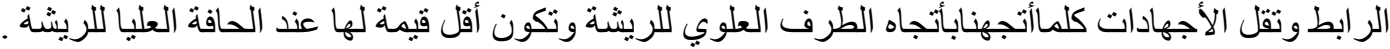

وتكون الأجهادات الناتجة عن قوة البخارقليلة مقارنة بالقوة الطاردة المركزية حيث تبلغ الأجهادات الناتجة عن القوة الطاردة المركزية عشرة أضعاف الأجهادات الناتجة عن البخار البخار

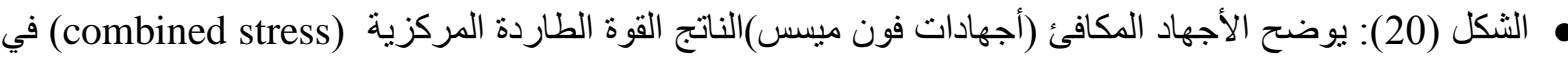

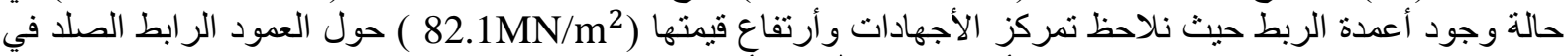

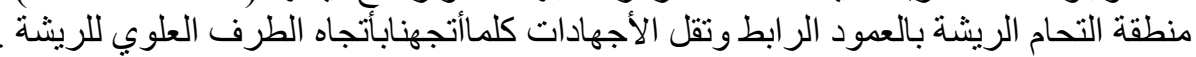

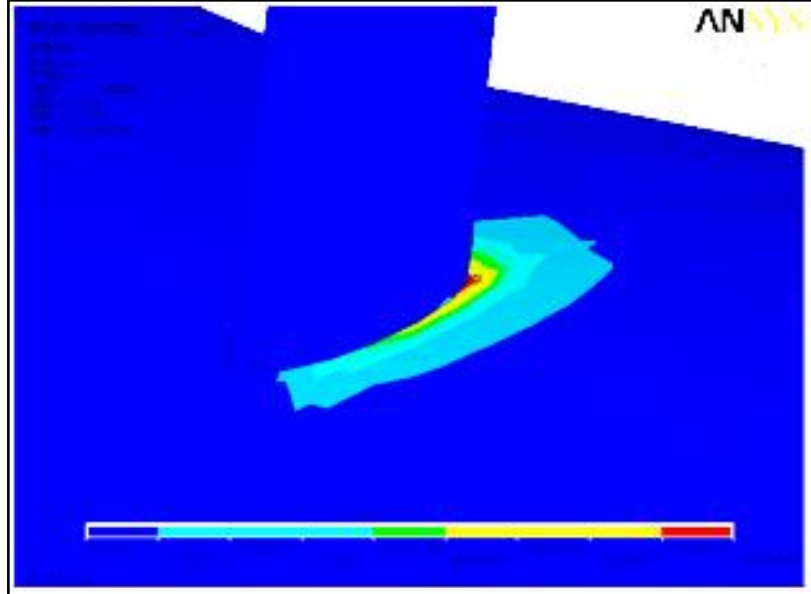

الثكل (20) : الاجهاد المكافئ الناتج عن قوة البخار في

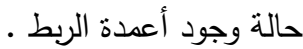

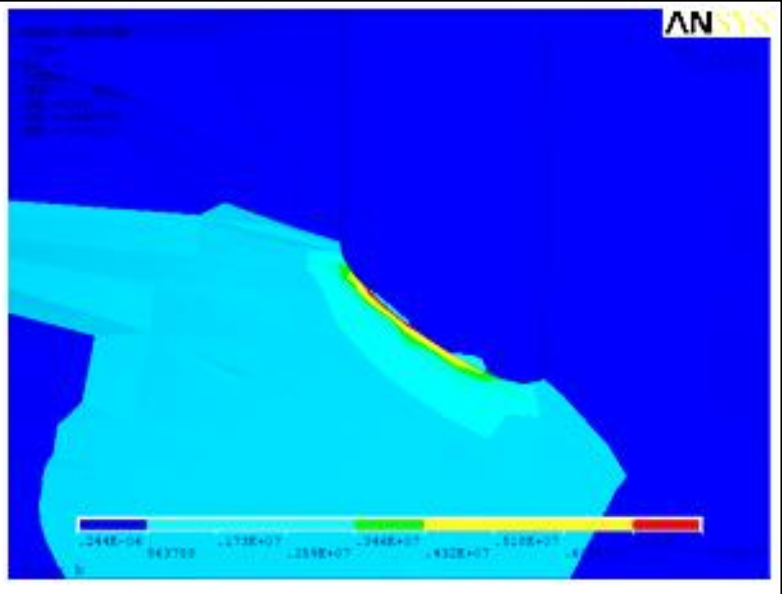

الثكل (19) : الاجهاد المكافئ الناتج عن الأجهاد

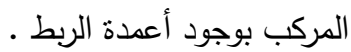

• توضح الاشكال (21) ،(22) (23) تغير قيم الأجهادات المكافئة الناتجة عن الاجهاد المركب و القوة الطاردة المركزية وقوة البخار في حالة وجود عمود الربط و عدم وجودها حيث نلاحظ في جميع الحالات قلة الأجهادات الناتجة على الريشة الرئة

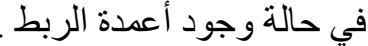

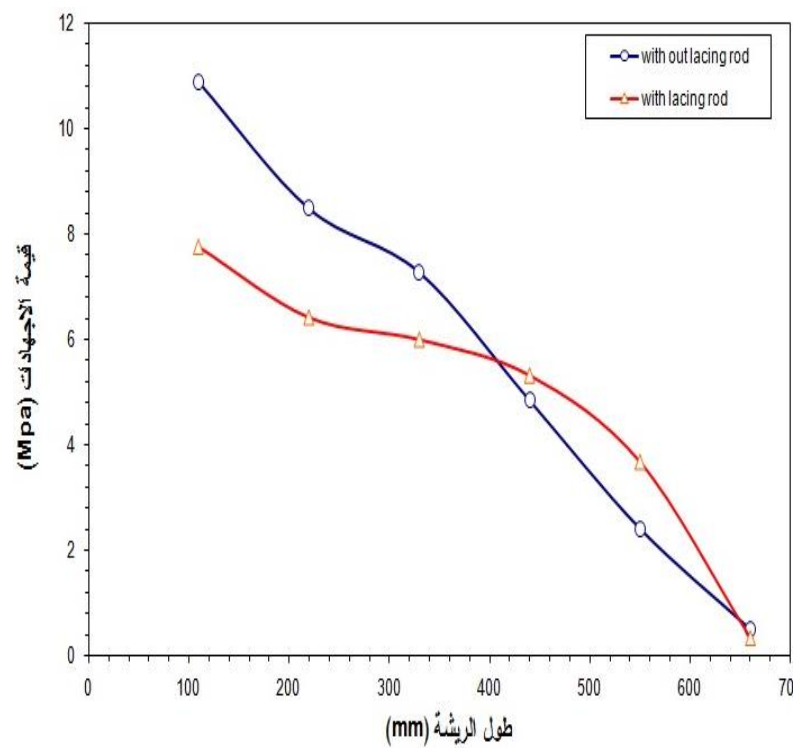

الثكل (22): تغير قيم الاجهاد المكافئ الناتج عن قوة

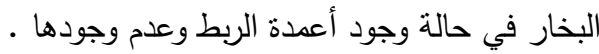

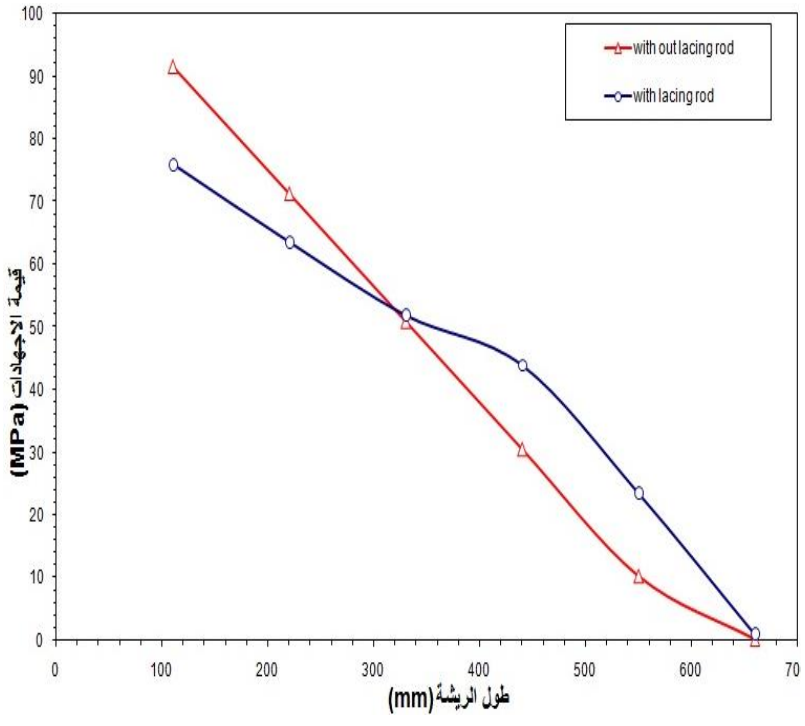

الثكل (21): تغير قيم الأجهاد المكافئ الناتج عن القوة الطاردة المركزية في حالة وجود أعمدة الربط وعدم وجودها ـ العائ. 


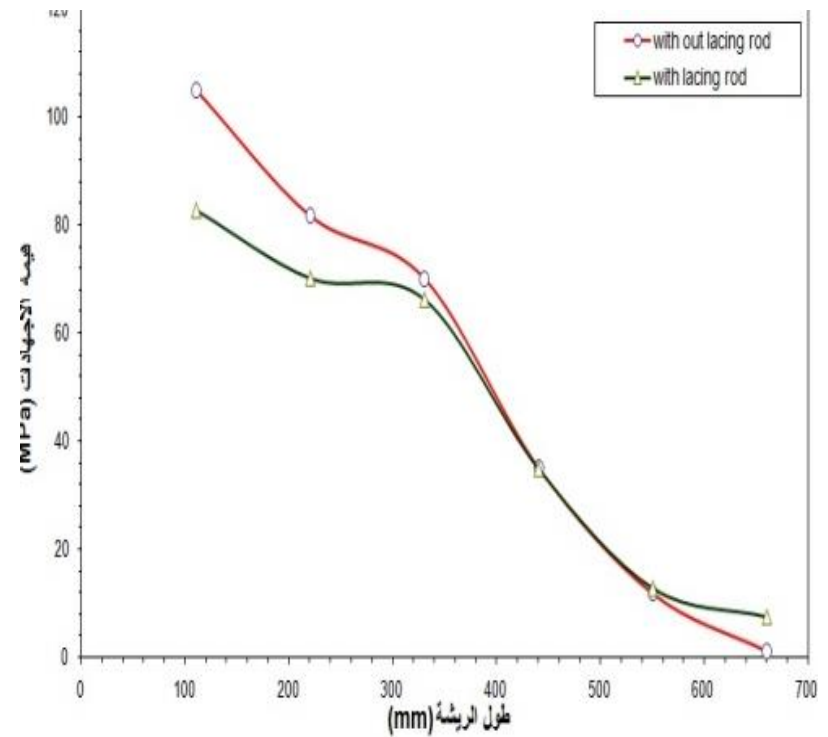

الشكل (23): تغير قيم الاجهاد المكافئ الناتج عن

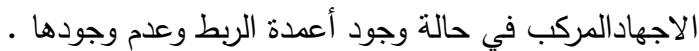

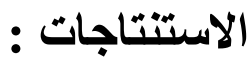

1. بالنسبة لموقع تكسر وفثل الريش تركزت حوادث الفثل في منطقة الجنيح و العمود الر ابط بنسة (70\%) وجذر الريشة نسبة الكسر (30\%) .

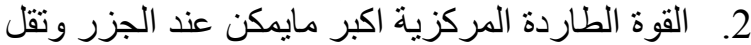

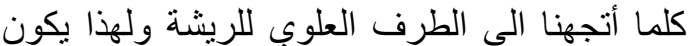

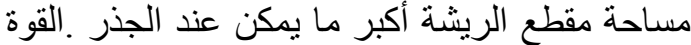

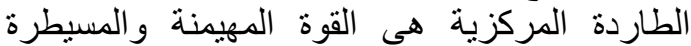
وتبلغ أضعاف القوة الناتجة عن البخار .القوة الطاردة التهاة

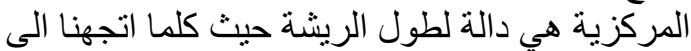
الطرف العلوي للريشة تقل القوة الرئة الطاردة المركزية

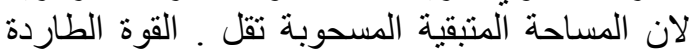

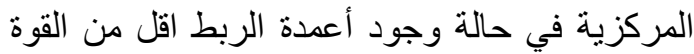

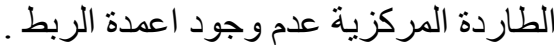

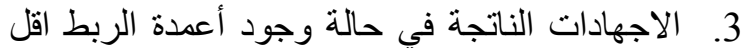

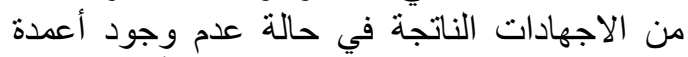
ربط ويعزى السبب في حالّة عدم وجود أعمدة الربط الإن

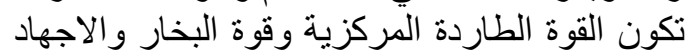

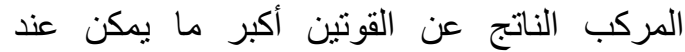

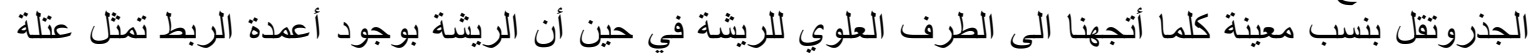

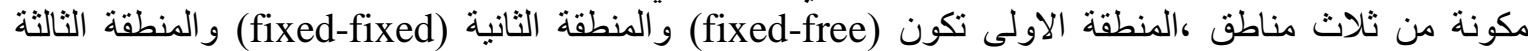
(fixed-free)

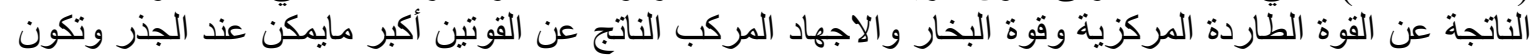

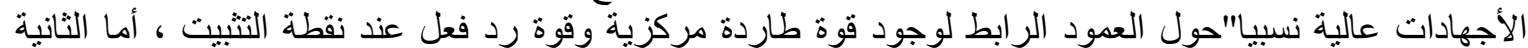

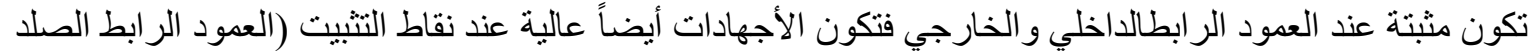

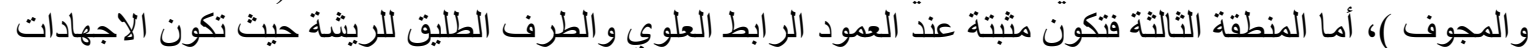

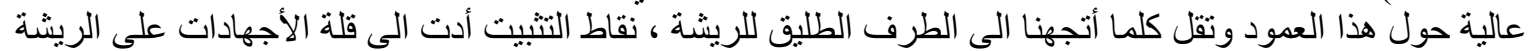

المصادر :

1. Church, E.F, "Steam Turbine" , London, McGraw-Hill, England, 1950.

2. Heinz p.bloch, murari p.singh, "steam Turbine", Mc Hill, England,(2009).

$$
\text { 3. القواعد الأساسية لمحطة كهرباء بيجي الحرارية أعداد المهندس وعد الله صبحي شاكر العراق، (2007) . }
$$

4. Mayer,K.H, and Besigk,C.," Influence of Design, "Manufacture and operation on useful life of corrosion Stressed Turbine blade" , work shop Proceeding of corrosion Fatigue of steam turbine Blade", Palo Alto, California, U.S.A.Sept 21-24(1980) .

5. الجبوري،عمر خليل احمد، "دراسة أسباب تكسر ريش المراحل الأخيرة للتوربينات البخارية في محطة بيجي

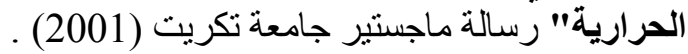

6. A. Thakker,H.B.khaleeq,F.Hourigan and J.Jarvis "stress Analysis of Impulse Turbine", Proceeding of the Elleventh International Offshore and Polar Engineering conference, ,Limerick, Ireland(2001).

7. Gary-steam Turbine Engineering, RWE npower Engineering (mechanical and Electrical Engineering power Industry), "Forces on Large Steam Turbine Blades" , England (2007). 
8. Arkan.K.HusainAl-Taie "Stress Evaluation of low-pressure steam TurbineBlade and Design of Reduce stress Blade" , Eng.Tech.Vol.26 No.2, Iraq (2008) .

9 .C.A. Poblano-Salas,J.D.OBarceinas-Sanchez, and J.C. Sanchez-Jimenez, "Failure analysis of an AISI 410 Stainless Steel airfoil in a steam turbine" Engineering Failure Analysis,vol18 68-74 Mexico(2011) .

10. T.D.EASTOP, A.MC.CONKEY "Applied Thermodynamics for Engineering Technologist", (1982).

11. Gordon J. Van Wylen " Fundamentals of classical Thermodynamic" fourth Edition, U.S.A. (1994) .

$$
\text { 12. AUTOCAD (2010) ، للكاتب جورج أمور ا . }
$$

13. James A. Leach "AUTOCAD 2007 INSTRUCTOR" A student Guide to complete coverage of Auto CAD commands and features, McGraw-Hill Graphics series, England, (2007) .

\section{Help of Auto CAD (2010) .}

15. Erdogan Madenci, IbrahimGuven " The Finite Element Method and Application in Engineering using Ansys" U.S.A(2006).

16. N.Nakasone, T.A.Stolarski and S.Yoshimoto ,"Engineering Analysis with Ansys software" Tokyo, Japan (2006).

\section{Help of ANSYS program V 12.1.}

تم اجراء البحث في كلية ألهندسة = جامعة ألموصل 